\title{
Determinants of weight gain in patients with depressive or bipolar disorders
}

\section{Abstract}

Introduction: Mental health disorders constitute a growing disease burden in France. In particular, hospitalized patients with depressive or bipolar disorders suffer from weight gain impairing adherence to treatment and the quality of life. The objective of this preliminary study is to better understand determinants of weight gain and their synergetic effects, with the ultimate aim to develop a screening tool identifying at risk patients.

Material and methods: Subjects were recruited at the private psychiatric clinic of Littoral, Rang-du-Fliers in the North of France according to pre-definite inclusion criteria. Data assessment has been based on 1) a retrospective case-control study using electronic medical records of depressive or bipolar patients $(\mathrm{n}=207) ; 2)$ a prospective observational study $(\mathrm{n}=20)$ using the medical records, and additional questionnaires at T0 (interview-administered) and T1 (self-administered) after an average length of hospital stay of 24.8 days $( \pm 6.3)$ and 3$)$ a cross-sectional study in depressive patients $(n=40)$ under psychotropic treatment at the day hospital, using the medical records and an interview-administered questionnaire. Descriptive and analytical statistics (Spearman rank correlations, binary logistic regression) were performed with SPSS statistics 17.0.

Results: The mean age of all patients was 49.3 years $( \pm 10.7)$. Patients' weight change after hospital stay varied in the retrospective study from $+0.5 \mathrm{~kg}( \pm 1.98)$ [min: $-5.4 \mathrm{~kg}$; max: $+8,4 \mathrm{~kg}$ ]. Patients at the day hospital had an mean psychotropic treatment duration of 7.8 years $( \pm 7.8)$ and gained on average $17.6 \mathrm{~kg}( \pm 10,01)$. Weight gain was particularly related to the use of tricyclic antidepressants, classic antipsychotics and atypical antipsychotics. All psychotropic drugs were significantly associated with a change in eating behavior $\left(r_{s}=0.37\right)$ which was significantly associated with weight gain $\left(r_{s}=0.40\right)$. All studies observed significant associations between weight gain and a lower socio-economic profile (low financial resources, few social contacts, low level of education), polymedication and a sedentary lifestyle with a higher tobacco consumption and less sleeping hours.

Discussion: This preliminary study confirms the complex interaction between psychotropic treatments, the modification of eating behavior which can be triggered by other determinants of weight gain in patients with depressive or bipolar disorders, such as tobacco consumption. The results provide new perspectives to create an innovative screening tool to improve the nutritional care in at risk patients. The development of such a tool is ongoing.

\section{Conflict of Interest}

There is no conflict of interest 